\section{Acute lung injury prediction score: derivation and validation in a population-} based sample

\author{
C. Trillo-Alvarez*, R. Cartin-Ceba*, D.J. Kor”, M. Kojicic*, R. Kashyap*, S. Thakur*, \\ L. Thakur*, V. Herasevich*, M. Malinchoc and O. Gajic*
}

ABSTRACT: Early recognition of patients at high risk of acute lung injury (ALI) is critical for successful enrolment of patients in prevention strategies for this devastating syndrome. We aimed to develop and prospectively validate an ALI prediction score in a population-based sample of patients at risk.

In a retrospective derivation cohort, predisposing conditions for ALI were identified at the time of hospital admission. The score was calculated based on the results of logistic regression analysis. Prospective validation was performed in an independent cohort of patients at risk identified at the time of hospital admission.

In a derivation cohort of $\mathbf{4 0 9}$ patients with ALI risk factors, the lung injury prediction score discriminated patients who developed $A L I$ from those who did not with an area under the curve (AUC) of $0.84(95 \% \mathrm{Cl} 0.80-0.89$; Hosmer-Lemeshow $p=0.60)$. The performance was similar in a prospective validation cohort of 463 patients at risk of ALI (AUC 0.84, 95\% Cl 0.77-0.91; HosmerLemeshow $p=0.88$ ).

ALI prediction scores identify patients at high risk for ALI before intensive care unit admission. If externally validated, this model will serve to define the population of patients at high risk for ALI in whom future mechanistic studies and ALI prevention trials will be conducted.

KEYWORDS: Acute lung injury, acute respiratory distress syndrome, population studies

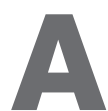

cute lung injury (ALI) and its more severe form acute respiratory distress syndrome (ARDS) are examples of critical care syndromes with limited treatment options once the condition is fully established. Pre-clinical studies support a "two-hit" model of ALI/ARDS development whereby exposure to pertinent risk factors modify the development and expression of ALI/ARDS in an already susceptible host with predisposing conditions [1]. The condition usually develops in patients with underlying risk factors (pneumonia, severe sepsis, trauma and aspiration) $[2,3]$ but is modified by different patients characteristics including genetic predisposition [4], as well as certain medical interventions (adverse ventilator settings and transfusion of alloimunised plasma) [5, 6]. Animal models provide compelling evidence in support of oxidative stress, lung deformation, loss of compartmentalisation of inflammation and intravascular coagulation as the pathogenic mechanisms involved in the development of ALI/ARDS [7-14]. However, many treatments targeting these mechanisms have failed to improve patient outcomes despite compelling pre-clinical data. It is probable that inadequate or delayed recognition and treatment of patients at risk of the full-blown syndrome have obscured the therapeutic window [15-19]. The recent National Institute of Health workshop [20] prioritised the development of strategies to perform ALI/ARDS prevention trials.

Importantly, epidemiological data suggest that ALI/ARDS is rarely present at the time of hospital admission. Rather, ALI/ARDS appears to develop over a period of hours to days in this subset of patients at risk [21-23]. Unfortunately, clinical studies are usually performed in the intensive care unit (ICU) setting, enrolling patients with established ALI/ARDS who are beyond the therapeutic window of potential prevention strategies. This delayed enrolment prevents adequate study of patients at risk.

A significant challenge with early enrolment of patients at risk of ALI/ARDS into prevention trials is the fact that the majority of patients with predisposing conditions never develop ALI/ ARDS and are never admitted to the ICU. This
AFFILIATIONS

Depts of *Internal Medicine,

${ }^{\#}$ Anesthesiology and the

Multidisciplinary Epidemiology and Translational Research in Intensive Care (M.E.T.R.I.C.) Program, and

"Health Sciences Research, Mayo Clinic College of Medicine, Rochester, MN, USA.

CORRESPONDENCE

0. Gajic

Dept of Internal Medicine

Mayo Clinic

200 First Street SW

Rochester

MN 55905

USA

E-mail: Gajic.0gnjen@mayo.edu

Received:

March 082010

Accepted after revision: June 022010

First published online:

June 182010

European Respiratory Journa

Print ISSN 0903-1936

Online ISSN 1399-3003 
makes the enrolment of unselected patients into ALI/ARDS prevention studies neither feasible nor efficient [21]. The likelihood of ALI development depends not only on specific risk factors (from 5\% with elective cardiopulmonary bypass [24] to $40 \%$ in patients with septic shock [25]), but also on the presence of specific risk modifiers. These include alcohol abuse [24-27], smoking [24, 27], hypoalbuminemia [28, 29], tachypnoea [25, 28], oxygen supplementation [30], chemotherapy [25, $31]$ and diabetes mellitus [28, 32].

To facilitate the enrolment of patients into future mechanistic and outcome studies, we aimed to develop and validate an ALI/ARDS prediction model which incorporates risk factors and risk modifiers that are present at the time of hospital admission, before the development of ALI. This model will serve to define populations of patients at high risk of ALI, and guide the interpretation of results and the satisfactory enrolment of patients into specific groupings for putative therapeutic interventions.

\section{MATERIALS AND METHODS}

This was an observational cohort study designed to develop and validate a numerical index which accurately estimates the probability of developing ALI/ARDS. The study was approved by the Institutional Review Board and all patients provided consent to the use of their medical records for research. The retrospective derivation cohort included Olmsted County (MN, USA) residents admitted to an adult ICU in Rochester (MN, USA) from January to December 2006. Exclusion criteria included age $<18$ yrs, pregnancy and a previous hospital admission during the study period. The prospective validation cohort included Olmsted County residents with risk factors (see below) for ALI/ARDS at the time of hospital admission admitted from November 2008 to May 2009. Exclusion criteria for the validation cohort included age $<18$ yrs, pregnancy and a previous admission during the study period. All patients were cared for at a single academic medical centre as it is the only hospital system with advanced ICU capabilities in this geographical area. Trained investigators abstracted data from the electronic medical records of patients from both cohorts and confirmed presence of specific ALI/ ARDS risk factors according to standardised definitions.

\section{Outcome variable}

The primary outcome of interest in this study was the development of ALI/ARDS during the hospital stay. Standard American-European Consensus Conference [33] criteria were used for determination of ALI/ARDS occurrence. Patients with possible ALI/ARDS were first identified with an electronic alert system (ALI sniffer). This system utilises a Microsoft SQL-based integrative database, ICU DataMart, where data are populated within $1 \mathrm{~h}$ of entry into the electronic medical record (EMR). An automatic alert was created if a patient had both the qualifying arterial oxygen tension/inspiratory oxygen fraction ratio on arterial blood gas analysis and a qualifying chest radiograph report; trigger words include "bilateral" and "infiltrate" or "oedema". This system has been validated in previous publications and has excellent sensitivity for identifying ALI/ARDS [34]. Records of alerted patients were independently reviewed by two trained investigators who underwent a structured ALI/ ARDS tutorial prior to reviewing the EMR in order to confirm presence of ALI/ARDS. Interobserver agreement was measured using Kappa values with disagreements solved by consensus.

\section{Predictor variables}

For model derivation, risk factors independently associated with development or prevention of ALI/ARDS in previously published studies were evaluated. These variables had to be recorded during the first $6 \mathrm{~h}$ of admission to the hospital to be considered to be present. Standardised definitions were used to identify risk factors (high risk trauma [28, 35-37], high-risk surgery [24, 38-40], aspiration [28, 35, 38, 41], sepsis [21, 35, 36, 38], shock [21, 42-44], pneumonia $[21,28,38,45]$ and pancreatitis $[1,21,46-50])$ and risk modifiers (alcohol abuse [24-27], smoking [24, 27], hypoalbuminemia [28, 29], tachypnoea [25, 28], oxygen supplementation [30], chemotheorapy [25, 31] and diabetes mellitus [28, 32]). The validation cohort included 467 Olmsted County patients who were admitted to hospital wards (ICU and non-ICU), excluding 1-day surgical procedures, cardiac observation, paediatric and maternity wards. Participants had to have at least one predisposing condition to be included in this validation cohort. The variables needed to generate the Lung Injury Prediction Study (LIPS) score were collected prospectively by trained study coordinators from the data recorded in the EMR during the first $6 \mathrm{~h}$ of admission.

\begin{tabular}{|c|c|c|}
\hline Patient characteristics & $\begin{array}{l}\text { Retrospective } \\
\text { derivation cohort }{ }^{\#}\end{array}$ & $\begin{array}{c}\text { Prospective } \\
\text { validation cohort }\end{array}$ \\
\hline \multicolumn{3}{|l|}{ Demographics } \\
\hline Age yrs & $68.0(57.0-78.0)$ & $68.0(51-84)$ \\
\hline Female & $186(45)$ & $243(52)$ \\
\hline \multicolumn{3}{|l|}{ Predisposing conditions } \\
\hline Sepsis & $47(11)$ & $257(55)$ \\
\hline Trauma & $18(4)$ & $27(6)$ \\
\hline Shock & $164(40)$ & $135(29)$ \\
\hline Pneumonia & $55(13)$ & $214(46)$ \\
\hline Aspiration & $19(5)$ & $44(9)$ \\
\hline Pancreatitis & $4(1)$ & $41(9)$ \\
\hline \multicolumn{3}{|l|}{ High-risk surgery } \\
\hline None & $308(75)$ & $419(90)$ \\
\hline Elective & $31(8)$ & $16(3)$ \\
\hline Emergent & $70(17)$ & $32(7)$ \\
\hline \multicolumn{3}{|l|}{ Risk modifiers } \\
\hline Alcohol & $26(6)$ & $36(8)$ \\
\hline Smoking & $175(43)$ & $198(42)$ \\
\hline Hypoalbuminaemia & $13(3)$ & $58(12)$ \\
\hline Diabetes & $87(21)$ & $116(25)$ \\
\hline Chemotherapy & $2(0)$ & $31(7)$ \\
\hline $\begin{array}{l}\text { Oxygen supplementation }>0.35 \\
\mathrm{Fl}_{1} \mathrm{O}_{2}^{+}\end{array}$ & $194(47)$ & $126(27)$ \\
\hline Tachypnoea $^{\S}$ & $51(12)$ & $47(10)$ \\
\hline
\end{tabular}

Data are presented as mean (interquartile range) or $\mathrm{n}(\%)$. Missing data smoking (83\% complete), alcohol (93\% complete) and serum albumin $(7 \%$ complete). Missing data were considered as negative. $\mathrm{Fl}_{1} \mathrm{O}_{2}$ : inspiratory oxygen fraction. ${ }^{*}$ : intensive care unit, $n=409 ;{ }^{\bullet}$ : hospital, $n=467 ;^{+}:>4 \mathrm{~L} \cdot \mathrm{min}^{-1}$ nasa cannula; ${ }^{\text {s. }}$ respiratory rate, 30 breaths $\cdot \mathrm{min}^{-1}$ 


\section{Statistical analyses}

14 previously reported independent predictors of ALI/ARDS were included in the model derivation. Seven of these predictors were considered predisposing conditions (high-risk trauma, high-risk surgery, sepsis, shock, pneumonia, aspiration and pancreatitis). The seven remaining predictors were considered modifier conditions (respiratory rate $>30$ breaths $\cdot \mathrm{min}^{-1}$, alcohol abuse, hypoalbuminemia, oxygen supplementation, chemotherapy, diabetes mellitus and smoking history). The relative weight assigned to each LIPS covariate was quantified according to the $\beta$-coefficients from logistic regression analysis in the derivation cohort. Consideration was given to the magnitude of effects reported in previous studies showing an independent association between a specific factor and ALI/ARDS. In order to assess the discriminatory power of our rule, the area under the receiver-operating curve (AUC) of the prediction scale was determined. The threshold score providing the best combination of sensitivity and specificity was determined by AUC analysis of the retrospective cohort. We evaluated the model for lack of fit with the Hosmer-Lemeshow statistic.

\begin{tabular}{|c|c|c|c|}
\hline Patient characteristics & ALI & No ALI & p-value \\
\hline Subjects $\mathrm{n}$ & 68 & 341 & \\
\hline \multicolumn{4}{|l|}{ Demographics } \\
\hline Age yrs & $68.0(19.0-96.0)$ & $68.0(18.0-97.0)$ & 0.69 \\
\hline Female & $32(47)$ & $154(45)$ & 0.77 \\
\hline \multicolumn{4}{|l|}{ Predisposing conditions } \\
\hline Sepsis & $14(21)$ & $33(10)$ & 0.01 \\
\hline Shock & $44(65)$ & $120(35)$ & $<0.001$ \\
\hline Trauma & $5(7)$ & $13(4)$ & 0.19 \\
\hline Pneumonia & $9(13)$ & $46(13)$ & 0.96 \\
\hline Aspiration & $7(10)$ & $12(4)$ & 0.015 \\
\hline Pancreatitis & $1(1)$ & $3(1)$ & 0.65 \\
\hline \multicolumn{4}{|l|}{ High-risk surgery } \\
\hline Elective & $8(12)$ & $23(7)$ & 0.15 \\
\hline Emergent & $25(37)$ & $52(15)$ & $<0.001$ \\
\hline \multicolumn{4}{|l|}{ Risk modifiers } \\
\hline Alcohol & $12(18)$ & $14(4)$ & $<0.001$ \\
\hline Smoking & $36(53)$ & $139(41)$ & 0.06 \\
\hline Hypoalbuminaemia & $7(10)$ & $6(2)$ & $<0.001$ \\
\hline Diabetes & $13(19)$ & $74(22)$ & 0.63 \\
\hline No sepsis & $10(19)$ & $56(18)$ & 0.95 \\
\hline Sepsis & $3(21)$ & $18(55)$ & 0.05 \\
\hline Chemotherapy & $1(1)$ & $1(0)$ & 0.20 \\
\hline $\begin{array}{l}\text { Oxygen supplementation } \\
>0.35^{\#}\end{array}$ & $46(68)$ & $148(43)$ & $<0.001$ \\
\hline Tachypnoea" & $18(26)$ & $33(10)$ & $<0.001$ \\
\hline
\end{tabular}

Data are presented as mean (interquartile range) or $n(\%)$, unless otherwise stated. Missing data: smoking (83\% complete), alcohol (93\% complete) and serum albumin (7\% complete). Missing data were considered as negative. ${ }^{\#}:>4 \mathrm{~L} \cdot \mathrm{min}^{-1}$ nasal cannula; ${ }^{\bullet}$ : respiratory rate, 30 breaths $\cdot \mathrm{min}^{-1}$.

\section{RESULTS}

The characteristics of both retrospective (ICU) and prospective (hospital) cohorts are presented in table 1 .

\section{Retrospective derivation cohort}

Of 409 patients with at least one predisposing condition at the time of hospital admission, out of 1,707 Olmsted County admissions who required ICU care during the study period, 68 (17\%) developed ALI/ARDS. Tables 2 and 3 provide univariate and multivariate comparisons of specific risk factors and risk modifiers in the derivation cohort. Corresponding LIPS points are shown in table 3 as well.

The LIPS model discriminated well between patients who did and did not develop ALI (AUC 0.84, 95\% CI 0.80-0.89) and was well calibrated (Hosmer-Lemeshow $\mathrm{p}=0.60$ ) (fig. 1a).

\section{Prospective validation cohort}

The validation cohort included 467 patients with at least one predisposing condition for ALI/ARDS identified at the time of hospital admission; out of 2,600 Olmsted County admissions screened during the study period. The incidence of ALI/ARDS was $7 \%$. Performance of the LIPS was similar to that observed in the retrospective derivation cohort with an AUC of 0.84 (95\% CI 0.77-0.91). The model was well calibrated (HosmerLemeshow $\mathrm{p}=0.88$ ) (fig. 1b).

Table 4 describes the performance of the score in both cohorts. Despite similar characteristics, positive predictive value was

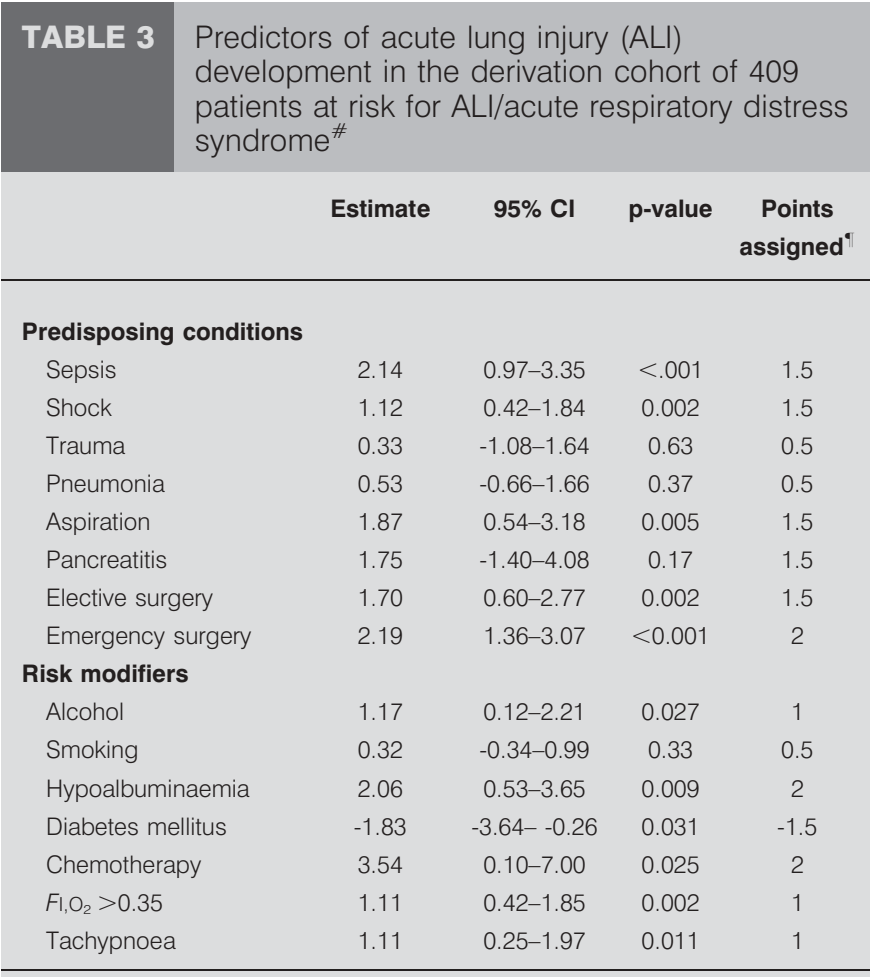

$\mathrm{Fl}_{1} \mathrm{O}_{2}$ : inspiratory oxygen fraction. ${ }^{\#}$ : parameter estimates from a multivariate analysis and corresponding Lung Injury Prediction Study points; ${ }^{\bullet}$ : if positive finding. 

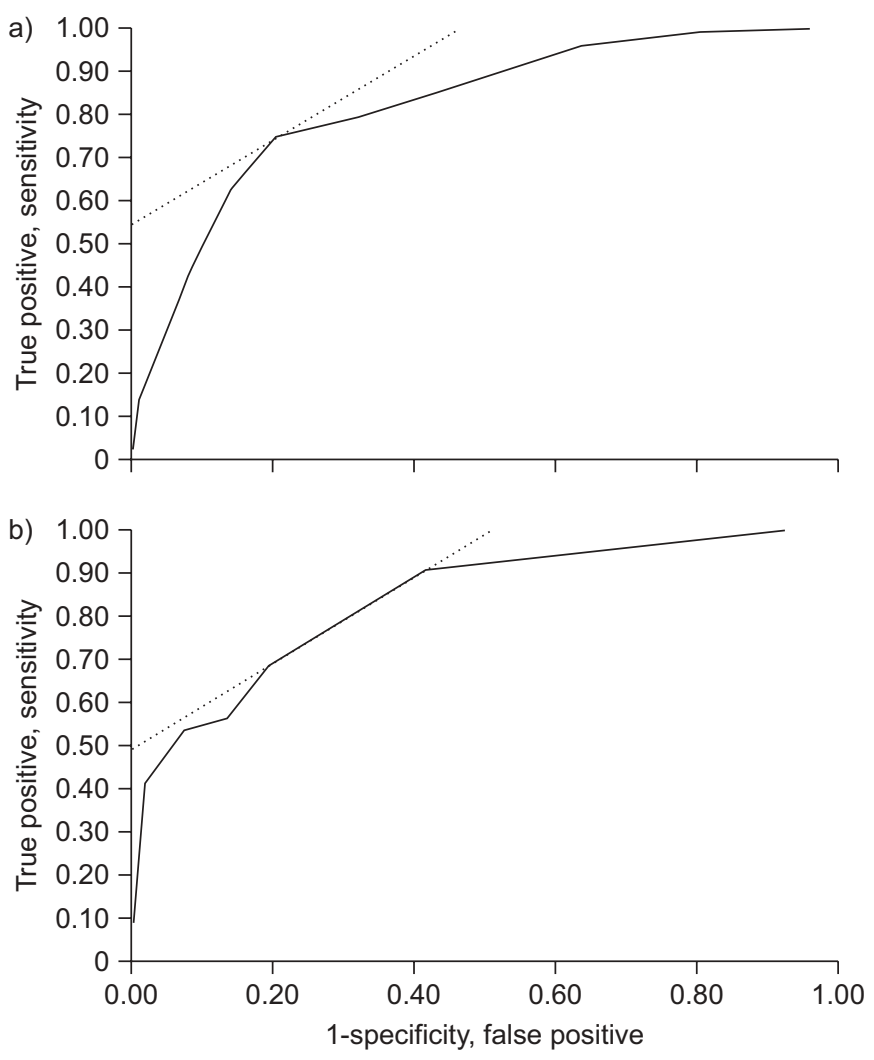

FIGURE 1. a) Receiver operating characteristic curve for acute lung injury (ALI) development in the derivation sample. The area under the receiver operating characteristic curve was $0.84(95 \% \mathrm{Cl}$ 0.80-0.89) and was well calibrated (HosmerLemeshow $p=0.601$ ). b) Receiver operating characteristic curve for ALI development in the validation sample. The area under the receiver operating characteristic curve was 0.84 (95\% $\mathrm{Cl} 0.77-0.91)$ and was well calibrated (Hosmer-Lemeshow $\mathrm{p}=0.881$ ).

lower in the validation cohort due to a lower incidence of ALI/ ARDS in a hospital (rather than ICU) based cohort.

\section{DISCUSSION}

In this population-based cohort we developed a prediction model for identifying patients at high risk of ALI/ARDS at the time of hospital admission. The model showed good discrimination and calibration in both the derivation and validation cohorts.
Importantly, when comparing the validation cohort (hospitalised patients regardless of ICU disposition at the time of admission) to the derivation cohort (only ICU patients), the proportion of patients with risk factors who developed ALI was markedly reduced. Similar results were recently published by FERGUSON et al. [21] where only $7 \%$ of hospitalised patients with sepsis, $2 \%$ with pancreatitis, $10 \%$ of patients with pneumonia and $15 \%$ of patients with witnessed aspiration developed ALI. Indeed, the majority of patients with predisposing conditions never develop ALI/ARDS and are never admitted to the ICU [21]. This makes the enrolment of unselected patients into ALI/ ARDS prevention studies neither feasible nor efficient without a method for identifying those who are at high risk. The failure to take into account multiple triggers that influence ALI/ARDS development has probably led to the discarding of a number of potentially important therapeutic advances in ARDS which may prove to be effective in specific and highly characterised groups of patients, particularly if applied early in the course of illness.

Our study has confirmed many but not all of the previously published ALI risk factors. In spite of a striking increase of incidence of ALI/ARDS in the elderly in the study by RUBENFIELD et al. [3], age did not predict ALI/ARDS development in our derivation cohort and, therefore, was not taken into account in the final model.

While some previous studies reported the increased risk of ALI/ARDS in the elderly [5, 27, 51], other studies have not confirmed this association [52-54]. It could be argued that elderly patients seem to have an increased incidence of ALI/ ARDS as they tend to have more sepsis, pneumonia and aspiration, and require more medical interventions. However, in patients admitted to the hospital with a risk factor (pneumonia or sepsis), age does not seem to increase the risk of ALI/ARDS development. Indeed, recent work implies that incidence of ALI/ARDS due to community-acquired pneumonia is lower in patients aged $\geqslant 85$ yrs [52].

Risk prediction models have been increasingly used to identify high-risk patients who may benefit from specific interventions. While their accuracy and precision are often limited, the models developed for severe pneumonia $[55,56]$ and perioperative cardiovascular complications [57] have been extensively used in both clinical practice and research. This study is the first attempt to develop a similar risk-prediction tool for the development of ALI/ARDS in patients at risk.

\section{TABLE 4 Performance of Lung Injury Prediction Study (LIPS) in the two cohorts}

Retrospective derivation cohort: ICU

Incidence of ALI/ARDS

AUC $(95 \% \mathrm{Cl})$

Sensitivity $(95 \% \mathrm{CI})$, LIPS $>3$

Specificity $(95 \% \mathrm{CI})$, LIPS $>3$

Positive predictive value $(95 \% \mathrm{CI})$, LIPS $>3$

Positive likelihood ratio $(95 \% \mathrm{CI})$, LIPS $>3$

Negative likelihood ratio $(95 \% \mathrm{CI})$, LIPS $>3$
$0.17(68 / 409)$

$0.84(0.80-0.89)$

$0.41(0.32-0.50)$

$0.90(0.89-0.92)$

$0.46(0.36-0.56)$

$4.26(2.77-6.41)$

$0.65(0.54-0.77)$

Prospective validation cohort: hospital

$0.07(32 / 463)$

$0.84(0.76-0.92)$

$0.69(0.53-0.82)$

$0.84(0.83-0.85)$

$0.24(0.18-0.28)$

$4.27(3.03-5.39)$

$0.37(0.22-0.56)$

ICU: intensive care unit; ALI: acute lung injury; ARDS: acute respiratory distress syndrome; AUC: area under the curve. 
The overall performance of the LIPS tool is modest and it is probable that the model could be refined by: 1) separating specific predisposing conditions (refining the high-risk surgery according to a surgery type: cardiovascular, thoracic or acute abdomen); 2) utilising sophisticated analytic methods such as neural network analysis and recursive partitioning; and 3) adding additional pertinent variables as their association with ALI/ARDS are described.

Nonetheless, the LIPS model efficiently discriminates the patients who have a small chance of developing ALI/ARDS (good specificity), while maintaining appropriate sensitivity as a screening tool. Through the early and accurate identification of patients at high risk of ALI/ARDS at the time of hospitalisation, the model will allow timely and efficient enrolment of patients into future ALI/ARDS mechanistic studies and prevention trials. If externally validated, this tool may also be used in clinical practice to alert providers of patients who are at particular risk of ALI/ARDS.

The LIPS model utilises variables that are clearly defined and routinely available in the medical record. It does not require testing beyond the standard of care and is not restricted to an ICU population. It identifies patients early, at the time of hospital admission, and is validated for hospitalised patients irrespective of their required intensity of care at the time of admission. The population-based sample increases generalisability by avoiding the referral bias often found in tertiary academic medical centres. However, the most important limitation of our study is the fact that, although population based, both cohorts come from a single institution with specific practice patterns, in addition to a suburban homogenous population. The small sample size poses a significant potential for over fitting the logistic regression model, and further refinement and validation is needed prior to clinical use of this tool. The US Critical Illness and Injury Trials Group are currently testing the external validity of the LIPS (NCT00889772).

In conclusion, we have developed and validated an efficient and effective prediction tool for evaluating the risk of ALI/ ARDS at the time of hospital admission. As the majority of patients with predisposing conditions never develop ALI and are never admitted to the ICU, our prediction model can facilitate the timely and efficient enrolment of patients into mechanistic and outcome studies, as well as future ALI prevention trials. Since ALI patients represent an aetiologically diverse group, focus should be on defining subgroups that could benefit from particular target therapies. Nevertheless, multicenter validation is required before large-scale screening projects are performed.

\section{CLINICAL TRIAL}

This study is registered at ClincalTrials.gov with identifier number NCT00889772.

\section{STATEMENT OF INTEREST}

None declared.

\section{REFERENCES}

1 Matthay MA, Zimmerman GA, Esmon C, et al. Future research directions in acute lung injury: summary of a National Heart,
Lung, and Blood Institute working group. Am J Respir Crit Care Med 2003; 167: 1027-1035.

2 Ware LB, Matthay MA. The acute respiratory distress syndrome. N Engl J Med 2000; 342: 1334-1349.

3 Rubenfeld GD, Caldwell E, Peabody E, et al. Incidence and outcomes of acute lung injury. N Engl J Med 2005; 353: 1685-1693.

4 Gong MN. Genetic epidemiology of acute respiratory distress syndrome: implications for future prevention and treatment. Clin Chest Med 2006; 27: 705-724.

5 Gajic O, Frutos-Vivar F, Esteban A, et al. Ventilator settings as a risk factor for acute respiratory distress syndrome in mechanically ventilated patients. Intensive Care Med 2005; 31: 922-926.

6 Khan H, Belsher J, Yilmaz M, et al. Fresh-frozen plasma and platelet transfusions are associated with development of acute lung injury in critically ill medical patients. Chest 2007; 131: 1308-1314.

7 Cho HY, Jedlicka AE, Clarke R, et al. Role of Toll-like receptor-4 in genetic susceptibility to lung injury induced by residual oil fly ash. Physiol Genomics 2005; 22: 108-117.

8 Welty-Wolf KE, Carraway MS, Miller DL, et al. Coagulation blockade prevents sepsis-induced respiratory and renal failure in baboons. Am J Respir Crit Care Med 2001; 164: 1988-1996.

9 Bhatia M, Moochhala S. Role of inflammatory mediators in the pathophysiology of acute respiratory distress syndrome. J Pathol 2004; 202: 145-156

10 Chow CW, Herrera Abreu MT, Suzuki T, et al. Oxidative stress and acute lung injury. Am J Respir Cell Mol Biol 2003; 29: 427-431.

11 Idell S. Coagulation, fibrinolysis, and fibrin deposition in acute lung injury. Crit Care Med 2003; 31: 213-220.

12 Tremblay L, Valenza F, Ribeiro SP, et al. Injurious ventilatory strategies increase cytokines and c-fos m-RNA expression in an isolated rat lung model. J Clin Invest 1997; 99: 944-952.

13 Oeckler RA, Hubmayr RD. Ventilator-associated lung injury: a search for better therapeutic targets. Eur Respir J 2007; 30: 1216-1226.

14 Matute-Bello G, Frevert CW, Martin TR. Animal models of acute lung injury. Am J Physiol Lung Cell Mol Physiol 2008; 3: 379-399.

15 Ketoconazole for early treatment of acute lung injury and acute respiratory distress syndrome: a randomized controlled trial. The ARDS Network. JAMA 2000; 283: 1995-2002.

16 Jepsen S, Herlevsen P, Knudsen P. Antioxidant treatment with Nacetylcyesteine during adult respiratory distress syndrome: a prospective randomized placebo controlled study. Crit Care Med 1992; 20: 819-923.

17 Meade MO, Jacka MJ, Cook DJ, et al. Survey of interventions for the prevention and treatment of acute respiratory distress syndrome. Crit Care Med 2004; 32: 946-954.

18 Wiedemann HP, Arroliga AC, Komara J, et al. Randomized, placebo-controlled trial of lisofylline for early treatment of acute lung injury and acute respiratory distress syndrome. Crit Care Med 2002; 30: 1-6.

19 Zeiher BG, Artigas A, Vincent J-L, et al. Neutrophil elastase inhibition in acute lung injury: results of the STRIVE study. Crit Care Med 2004; 32: 1695-1702.

20 Spragg RG, Bernard GR, Checkley W, et al. Beyond mortality: future clinical research in acute lung injury: an NHLBI Workshop Report. Am J Respir Crit Care Med 2010; 181: 1121-1127.

21 Ferguson ND, Frutos-Vivar F, Esteban A, et al. Clinical risk conditions for acute lung injury in the intensive care unit and hospital ward: a prospective observational study. Crit Care 2007; 11: R96.

22 Greer R. The temporal evolution of acute respiratory distress syndrome following shock. Eur J Anaesthesiol 2010; 27: 226-232.

23 Yilmaz M, Keegan MT, Iscimen R, et al. Toward the prevention of acute lung injury: protocol-guided limitation of large tidal volume ventilation and inappropriate transfusion. Crit Care Med 2007; 35: $1660-1666$. 
24 Fernandez-Perez ER, Sprung J, Afessa B, et al. Intraoperative ventilator settings and acute lung injury after elective surgery: a nested case-control study. Thorax 2009; 64: 121-127.

25 Iscimen R, Cartin-Ceba R, Yilmaz M, et al. Risk factors for the development of acute lung injury in patients with septic shock: an observational cohort study. Crit Care Med 2008; 36: 1518-1522.

26 Moss M, Bucher B, Moore FA, et al. The role of chronic alcohol abuse in the development of acute respiratory distress syndrome in adults. JAMA 1996; 275: 50-54.

27 Iribarren C, Jacobs DR Jr, Sidney S, et al. Cigarette smoking, alcohol consumption, and risk of ards: a 15-year cohort study in a managed care setting. Chest 2000; 117: 163-168.

28 Gong MN, Thompson BT, Williams P, et al. Clinical predictors of and mortality in acute respiratory distress syndrome: potential role of red cell transfusion. Crit Care Med 2005; 33: 1191-1198.

29 Mangialardi RJ, Martin GS, Bernard GR, et al. Hypoproteinemia predicts acute respiratory distress syndrome development, weight gain, and death in patients with sepsis. Ibuprofen in Sepsis Study Group. Crit Care Med 2000; 28: 3137-3145.

30 Levitt JE, Bedi H, Calfee CS, et al. Identification of early acute lung injury at initial evaluation in an acute care setting prior to the onset of respiratory failure. Chest 2009; 135: 936-943.

31 Naito $Y$, Tsuchiya S, Ishihara S, et al. Impact of preexisting pulmonary fibrosis detected on chest radiograph and $\mathrm{CT}$ on the development of gefitinib-related interstitial lung disease. Am J Clin Oncol 2008; 31: 340-344.

32 Moss M, Guidot DM, Steinberg KP, et al. Diabetic patients have a decreased incidence of acute respiratory distress syndrome. Crit Care Med 2000; 28: 2187-2192.

33 Bernard GR, Artigas A, Brigham KL, et al. The AmericanEuropean Consensus Conference on ARDS. Definitions, mechanisms, relevant outcomes, and clinical trial coordination. Am J Respir Crit Care Med 1994; 149: 818-824.

34 Herasevich V, Yilmaz M, Khan $\mathrm{H}$, et al. Rule base system for identification of patients with specific critical care syndromes: the "sniffer" for acute lung injury. AMIA Annu Symp Proc 2007; 11: 972.

35 Pepe PE, Potkin RT, Reus DH, et al. Clinical predictors of the adult respiratory distress syndrome. Am J Surg 1982; 144: 124-130.

36 Hudson LD, Milberg JA, Anardi D, et al. Clinical risks for development of the acute respiratory distress syndrome. Am J Respir Crit Care Med 1995; 151: 293-301.

37 Derdak S. Acute respiratory distress syndrome in trauma patients. J Trauma 2007; 62: Suppl. 6, S58.

38 Fowler AA, Hamman RF, Good JT, et al. Adult respiratory distress syndrome: risk with common predispositions. Ann Intern Med 1983; 98: 593-597.

39 Arozullah AM, Daley J, Henderson WG, et al. Multifactorial risk index for predicting postoperative respiratory failure in men after major noncardiac surgery. The National Veterans Administration Surgical Quality Improvement Program. Ann Surg 2000; 232: 242-253.
40 Arozullah AM, Khuri SF, Henderson WG, et al. Development and validation of a multifactorial risk index for predicting postoperative pneumonia after major noncardiac surgery. Ann Intern Med 2001; 135: 847-857.

41 Marik PE. Aspiration pneumonitis and aspiration pneumonia N Engl J Med 2001; 344: 665-671.

42 Antonelli M, Levy M, Andrews PJ, et al. Hemodynamic monitoring in shock and implications for management. International Consensus Conference, Paris, France, 27-28 April 2006. Intensive Care Med 2007; 33: 575-590.

43 Rivers E, Nguyen B, Havstad S, et al. Early goal-directed therapy in the treatment of severe sepsis and septic shock. N Engl J Med 2001; 345: 1368-1377.

44 Gutierrez G, Reines HD, Wulf-Gutierrez ME. Clinical review: hemorrhagic shock. Crit Care 2004; 8: 373-381.

45 Hudson LD, Steinberg KP. Epidemiology of acute lung injury and ARDS. Chest 1999; 116: Suppl. 1, 74S-82S.

46 Napolitano LM. Pulmonary consequences of acute pancreatitis: critical role of the neutrophil. Crit Care Med 2002; 30: 2158-2159.

47 Pastor CM, Matthay MA, Frossard JL. Pancreatitis-associated acute lung injury: new insights. Chest 2003; 124: 2341-2351.

48 Lankisch PG, Rahlf G, Koop H. Pulmonary complications in fatal acute hemorrhagic pancreatitis. Dig Dis Sci 1983; 28: 110-116.

49 Renner IG, Savage WT 3rd, Pantoja JL, et al. Death due to acute pancreatitis. A retrospective analysis of 405 autopsy cases. Dig Dis Sci 1985; 30: 1005-1018.

50 Steer ML. Relationship between pancreatitis and lung diseases. Respir Physiol 2001; 128: 13-16.

51 Miller PR, Croce MA, Kilgo PD, et al. Acute respiratory distress syndrome in blunt trauma: identification of independent risk factors. Am Surg 2002; 68: 845-850.

52 Toba A, Yamazaki M, Mochizuki H, et al. Lower incidence of acute respiratory distress syndrome in community-acquired pneumonia patients aged 85 years or older. Respirology 2010; 15: 319-325.

53 Hughes CG, Weavind L, Banerjee A, et al. Intraoperative risk factors for acute respiratory distress syndrome in critically ill patients. Anesth Analg 2010; 111: 464-467.

54 TenHoor T, Mannino DM, Moss M. Risk Factors for ARDS in the United States: analysis of the 1993 National Mortality Followback Study. Chest 2001; 119: 1179-1184.

55 Fine MJ, Auble TE, Yealy DM, et al. A prediction rule to identify low-risk patients with community-acquired pneumonia. $N$ Engl J Med 1997; 336: 243-250.

56 Buising KL, Thursky KA, Black JF, et al. A prospective comparison of severity scores for identifying patients with severe community acquired pneumonia: reconsidering what is meant by severe pneumonia. Thorax 2006; 61: 419-424

57 Goldman L, Caldera DL, Nussbaum SR, et al. Multifactorial index of cardiac risk in noncardiac surgical procedures. N Engl J Med 1977; 297: 845-850. 